\title{
CAPITULO 93
}

\section{USO DA VISCOSSUPLEMENTAÇÃO NO TRATAMENTO DA ARTROCENTESE: REVISÃO DE LITERATURA}

$\underline{\text { DOI 10.4322/978-65-995353-2-1.c93 }}$

\begin{abstract}
Arthur Mendes Lima ${ }^{1}$, Thalita Oliveira da Silva Borba ${ }^{2}$, Anne Caroline Silva Freire de Sá ${ }^{3}$, André Almeida Antunes ${ }^{4}$, Erick Lúcio Souza Santos ${ }^{5}$, Fernanda Maria Matos Aragão de
\end{abstract} Souza $^{6}$, Jadson da Silva Santana ${ }^{7}$, Letícia Rafaella Marinho Honorato ${ }^{8}$, Alícia Lima de Lira ${ }^{9}$, Eduardo Stehling Urbano ${ }^{10}$

\author{
1arthurmlima_@hotmail.com - Universidade Federal de Juiz de Fora/UFJF \\ ${ }^{2}$ thalita.borba00@gmail.com - Centro Universitário Tabosa de Almeida ASCES/UNITA \\ ${ }^{3}$ annecarolinefreiree@gmail.com - Centro Universitário Tabosa de Almeida ASCES/UNITA \\ ${ }^{4}$ andredemu@yahoo.com.br - Centro Universitário de Belo Horizonte/Unibh \\ 5 ericklucioel@gmail.com - Centro Universitário Tabosa de Almeida ASCES/UNITA \\ ${ }^{6}$ aragaofernanda22@gmail.com - Graduada em Odontologia, pelo Centro Universitário Tabosa de \\ Almeida Asces/UNITA \\ 7jadsonufpe@gmail.com - Universidade Federal de Pernambuco/UFPE \\ ${ }^{8}$ leticiamarinhoh@ hotmail.com - Centro Universitário Tabosa de Almeida ASCES/UNITA \\ ${ }^{9}$ alicialimadl@gmail.com - Centro Universitário Tabosa de Almeida ASCES/UNITA \\ 10 esurss@ yahoo.com.br - Universidade Federal de Juiz de Fora /UFJF
}

\section{RESUMO}

Objetivo: Analisar a eficácia da artrocentese associada à viscossuplementação, ressaltando os seus benefícios, através de uma revisão de literatura. Método: Trata-se de uma revisão de literatura realizada a partir da inserção de descritores específicos nas bases de dados PubMed, SciELO e MedLine, onde foram incluídos estudos publicados na íntegra, entre 2010 a 2021, na língua portuguesa e inglesa, sendo excluídos resumos publicados em anais, textos incompletos, periódicos pagos, revisões e trabalhos sem fundamentação científica. A partir disso, foram selecionados 16 artigos que se relacionavam com o tema em questão. Resultados: A partir da inserção de descritores encontrou-se 128 estudos, os quais, após a filtragem por ano, idioma, gratuidade e texto 
completo disponível findaram-se em apenas 54. A partir da leitura dos títulos e resumos foram selecionados os artigos considerados de interesse para o objetivo do presente estudo que totalizaram 16 artigos. Conclusão: Pode-se concluir que a disfunção temporomandibular implica na qualidade de vida do paciente devido aos sintomas frequentemente divulgados. Diante disso, pode-se elaborar o tratamento cirúrgico ou conservador.

Palavras-chave: Artrocentese da Articulação Temporomandibular; Articulação Temporomandibular; Viscossuplementação;

Área Temática: Ciências da Saúde

E-mail do autor principal: arthurmlima_@hotmail.com

\section{INTRODUÇÃ̃o}

A disfunção temporomandibular (DTM) abrange problemas clínicos que interferem na articulação temporomandibular (ATM), músculos mastigatórios e estruturas anatômicas associadas à região. Os motivos relacionados à DTM são traumas diretos ou indiretos, estresse cumulativo e repetitivo, alterações na coluna, postura inadequada e o porte ergonômico da cabeça, além de fatores psicológicos. Pode-se incluir também, hábitos parafuncionais como apertamento dentário e bruxismo que implicam nas condições anatômicas e funcionais do sistema estomatognático (MACIEL, LANDIM e VASCONCELOS, 2018). Dentre os sintomas causados, são relatados dor facial, disfunção da mandíbula, sensibilidade e rigidez nos músculos da mastigação. Esses sintomas estão atrelados à disfunção da ATM (STEPAN, SHAW e OUE, 2017). De acordo com a literatura, a prevalência da disfunção temporomandibular é oscilante, atingindo cerca de 5-70 \% da população, sendo a dor o sintoma dominante em 40 por cento dos pacientes. Foi relatado que 40-75 \% da população apresenta um sinal de DTM e 33 por cento exibem um sintoma dessa disfunção (MACIEL, LANDIM e VASCONCELOS, 2018; SILVA e SOARES, 2018; STEPAN, SHAW e OUE, 2017). O acometimento, em especial, engloba indivíduos jovens e de meia-idade, com uma tendência maior ao gênero feminino. Em uma proporção que pode alterar, segundo a literatura, de 3:1 a 9:1 quando comparada aos homens (URBANO, et al.,2020; STEPAN, SHAW e OUE, 2017). A etiologia é regularmente multifatorial e, por isso, encontram-se diferentes intervenções. Nesse tocante, o objetivo do tratamento é uma melhora ou eliminação da dor e um aumento da função (BOULOUX et al., 2017).

O diagnóstico de disfunção temporomandibular pode ser difícil, uma vez que a DTM exibe uma variedade de sintomas, no qual não são exclusivos da disfunção temporomandibular 
(STEPAN, SHAW e OUE, 2017). Diante disso, os métodos diagnósticos para a identificação da DTM consiste em Diagnostic Criteria for Temporomandibular Disorders (DC/TMD), que se baseia num questionário publicado em 2014, a partir da adaptação do Research Diagnostic Criteria for TMD (RDC/TMD). O DC/TMD é distinguido em dois eixos, sendo o Eixo I utilizado com a finalidade de classificação em presença ou não de DTM, além da classificação do tipo de desordem; ao mesmo tempo que no Eixo II analisa aspectos psicossociais e comportamentais do paciente relacionado à DTM (SCHIFFMAN et al., 2014). Além disso, o reconhecimento da disfunção normalmente é clínico, sendo imprescindível os exames imaginológicos, essencialmente nos casos em que o tratamento cirúrgico é uma opção (DANTAS et al., 2019).

Para o tratamento das disfunções internas da ATM são sustentadas pela literatura as intervenções conservadoras, como o repouso funcional, anti-inflamatórios não esteroides (AINES), dispositivos interoclusais, exercícios fisioterápicos de suporte, injeções intra-articulares de corticosteróide; e terapias cirúrgicas, sendo estas variando desde cirurgias abertas até cirurgias consideradas minimamente invasivas, como a artrocentese e artroscopia da ATM (BONOTTO et al., 2011). A artrocentese não é indicada em casos de fibroanquilose, doenças intra-articulares como alterações ósseas e perfuração do disco articular. Usualmente, o protocolo primário de controle das DTM preconiza as medidas mais simples, reversíveis e menos invasivas. Entretanto, o tratamento conservador em determinados casos pode ser ineficaz, especialmente nos casos de distúrbios intra-articulares, sendo indicado as terapias cirúrgicas (DE RIU et al., 2013; URBANO etal.,2020).

Desse modo, a artrocentese relacionada a viscossuplementação é uma alternativa para casos em que a terapia conservadora não é bem vista. A viscossuplementação, corresponde na aplicação intra-articular de ácido hialurônico (AH), no intuito de diminuir o estresse da cartilagem articular através da lubrificação, e por obter mecanismos analgésicos e anti-inflamatórios, bloqueiam receptores e substâncias álgicas nos tecidos sinoviais, acarretando na recuperação da função fisiológica do líquido sinovial (BONOTTO et al., 2011). Com isso, a viscossuplementação pode ser vinculada a artrocentese que é um procedimento fácil, minimamente invasivo e altamente eficiente, tendo como objetivo de limpar a articulação de restos de tecidos, sangue e mediadores inflamatórios, além de aumentar a amplitude de abertura da boca em pacientes com restrição de movimentos mandibulares (LEE et al., 2013). 
Diante disso, o objetivo do trabalho é analisar a eficácia da artrocentese associada à viscossuplementação, através de uma revisão de literatura.

\section{MÉTODO}

Trata-se de uma revisão de literatura feita nas bases de dados PubMed, SciELO e MedLine realizada a partir do cruzamento dos descritores "Artrocentese da Articulação Temporomandibular; Articulação Temporomandibular; Viscossuplementação"; e seus correspondentes em inglês, entre os anos de 2010 a 2021, utilizando o operador booleano "AND". Foram incluídas revisões de literatura, relatos de caso, meta análises e ensaios clínicos. Além disso, foram excluídos resumos publicados em ANAIS, artigos incompletos, periódicos pagos ou trabalhos sem fundamentação científica. Frente a isso, foram encontrados 54 artigos, e selecionados 16 que se relacionavam com o tema em questão.

\section{RESULTADOS E DISCUSSÃO}

Silva e Soares (2018) e Azato et al. (2013) relataram que o aparecimento e perpetuação da DTM estão relacionados à interação de fatores, como estresse, lassidão ligamentar, trauma, hábitos parafuncionais, alterações sistêmicas e mudanças internas e externas na estrutura da articulação temporomandibular, sendo a DTM responsável pela causa de dor crônica da região orofacial, onde, dentre os indivíduos afetados, somente $12 \%$ possuem sintomas graves o suficiente para procurar tratamento. Não obstante, Silva e Soares (2018) apontam que para a realização do diagnóstico da disfunção temporomandibular, diversos questionários, índices e critérios diagnósticos devem ser utilizados adjunto com o exame clínico, onde, o critério diagnóstico em pesquisa para DTM (RDC/TMD) é apontado como uma das melhores ferramentas estruturadas para o diagnóstico da DTM. Além disso, o uso de exames de imagem que auxiliem a complementar o diagnóstico podem ser usados, como radiografia panorâmica, tomografia convencional da ATM, tomografia computadorizada da ATM, artrografia da ATM e a ressonância magnética nuclear, onde as imagens geradas, proporcionam a realização de vários cortes na imagem captada, permitindo a visão por 
ângulos

diversos.

Stepan, Shaw e Oue (2017), afirmam que o tratamento é determinado pela sintomatologia. No entanto, pode ser difícil determinar se a dor do músculo facial é primária ou resultado de disfunção da articulação intra-articular. Em geral, a mialgia e a disfunção articular ocorrem em conjunto. A orientação do paciente é um primeiro passo essencial na conduta. O tratamento se baseia numa alimentação leve, realização de compressas térmicas no local da dor e técnicas de relaxamento. Nesse contexto, Lee et al. (2013), condizem da mesma linha de raciocínio, destacando que o tratamento da DTM pode ser fragmentado em duas categorias sendo, o método conservador e o método cirúrgico. A conduta conservadora inclui medicamentos, fisioterapia, terapia com talas e diretrizes quanto aos hábitos parafuncionais. Os tratamentos cirúrgicos têm por sua vez, artrocentese, artroplastia, discectomia e reconstrução da ATM.

A artrocentese possibilita técnicas diferentes conforme a literatura, como a cânula única de Shepard, unidade concêntrica de agulhas, cânula dupla de agulhas, agulha única e a técnica de duas agulhas (GROSSMAN, 2012). Nesse contexto, o objetivo da artrocentese é remover mediadores inflamatórios, como interleucinas, radicais livres e restos celulares além de eximir aderências entre o disco e a fossa articular através da limpeza do compartimento superior da ATM com manipulação simultânea da mandíbula (ROSSINI et al., 2021). Esse procedimento pode ser realizado isoladamente ou associado a injeções intra-articulares de medicamentos (URBANO, et al., 2020; BOULOUX et al., 2017).

Os medicamentos com possibilidade de uso nas injeções intra-articulares são os corticoesteroides e o hialuronato de sódio (HS), sendo este último estipulado como viscossuplementação. No caso da viscossuplementação, o AH tem por característica potencial de lubrificação da ATM, além de desempenhar propriedades analgésica, anti-inflamatória e condroprotetora (URBANO, et al., 2020; ROSSINI et al., 2021). Num estudo comparativo de Giraddi et al., 2012) com relação ao uso da AH e corticosteróides após a intervenção com artrocentese, conclui-se que não existe distinção significativa entre esses medicamentos em relação à redução da dor e aumento da abertura bucal, e que ambos mostram excelentes resultados no tratamento. Entretanto, existe uma observação em relação ao uso de corticosteróides intraarticulares, visto que na literatura há relatos de que este fármaco pode causar efeitos colaterais a longo prazo, como destruição da cartilagem articular, reabsorção óssea e infecções (ABDULRAZZAK et al., 2021; GIRADDI et al., 2012). 
O maior componente do líquido sinovial é o AH, sendo relevante em função da lubrificação dos tecidos articulares, por causa do seu alto peso molecular. Nos processos inflamatórios e degenerativos das articulações a concentração e o peso molecular do AH estão reduzidos. A injeção do HS aumenta a quantidade e o peso molecular de AH no líquido sinovial, o que reflete no alívio da dor. Ou seja, a desobstrução das zonas de aderências entre o disco articular e a fossa mandibular amplificam a mobilidade articular, permitindo melhor circulação do líquido sinovial. Portanto, esses mecanismos de ação favorecem o avanço do quadro obtido pela paciente, após o tratamento com ambas as técnicas. A melhora na abertura bucal da paciente está relacionada com a eliminação de adesões, alívio da pressão negativa sobre o disco articular e recuperação do disco para a posição original durante a lavagem, o que também gera uma melhora dos ruídos articulares (BONOTTO et al., 2011). Em resumo, o AH proporciona aumento da lubrificação articular por ampliar a viscosidade do líquido sinovial, na qual exerce função protetora de choque, conservação da homeostase, proporcionando a ativação de processos de reparo e regulação das ações que interferem na síntese do líquido sinovial de células endógenas, provocando assim, uma maior mobilidade articular, diminuindo o atrito e ruído favorecendo a perfusão de nutrientes e metabólitos do líquido sinovial para os tecidos vasculares (ROSSINI et al., 2021).

Diante dessas técnicas, existe a chance de complicações como paralisia do ramo zigomático e temporal do nervo facial provocada pelo bloqueio anestésico local ou pelo próprio edema; paralisia zigomática ou de ramo bucal devido a trauma de agulha; edema pós-operatório causado por vazamento de solução intra-articular; hematoma periauricular; sangramento perioperatório por lesão vascular; bradicardia, fratura de instrumentos dentro da articulação e perfuração intracranial (GROSSMANN, 2012; MATSA et al., 2010; TAN e KRISHNASWAMY, 2012). Outrossim, Grossman (2012) aponta que outras complicações são possíveis como a formação de microhemorragias extra e intra-articulares, que podem avançar para anquilose fibrótica da ATM e até mesmo dores de difícil manuseio, como síndrome dolorosa regional complexa tipo I. No caso da viscossuplementação as principais intercorrências são reações no local da injeção da medicação, que se mostra como dor e edema (URBANO, et al., 2020).

Bouloux et al. (2017) evidenciaram que a artrocentese isolada é eficaz na redução da dor em pacientes com artralgia ou distúrbio interno, uma vez que diminui o nível de citocinas inflamatórias e proteínas degradadas na ATM, resultando em uma diminuição paralela da dor. Todavia, Lee et al. (2013) apontam que a artrocentese associada à viscossuplementação é um 
método eficiente no tratamento de DTM, devido aos seus mecanismos fisiológicos condroprotetor, anti-inflamatório e analgésicos; além de serem consideradas técnicas seguras. Outrossim, a associação dessas técnicas com placas neuromioelaxantes potencializam ainda mais o tratamento, ou seja, a combinação da terapia conservadora com a injeção de $\mathrm{AH}$ mais a artrocentese tem resultados significativos. Dessa maneira, pode-se dizer que a DTM apresenta diferentes caminhos a serem seguidos no quesito plano de tratamento e à associação da artrocentese com a viscossuplementação oferece benefícios mais expressivos quando comparada a artrocentese isolada. Perante a isso, o operador junto com sua equipe decidirá qual o melhor caminho a ser traçado para o sucesso no tratamento.

\section{CONCLUSÃO}

Pode-se concluir que a disfunção temporomandibular pode causar limitações e diminuição da qualidade de vida das pessoas por conta do quadro álgico doloroso comumente referido como sintoma. Sendo assim, pode-se traçar o plano de tratamento conservador ou cirúrgico,visto que este último engloba a artrocentese isolada ou associada a corticosteroides ou a injeção de ácido hialurônico, sendo que a injeção de $\mathrm{AH}$ oferece um método eficaz para o tratamento das desordens temporomandibulares por proporcionar aumento da lubrificação articular ampliando a viscosidade do líquido sinovial. 


\section{REFERÊNCIAS}

ABDULRAZZAK, N. J. et al. Arthrocentesis versus glucocorticosteroid injection for internal derangement of temporomandibular joint. Oral And Maxillofacial Surgery, v. 25, n. 2, p. 191197, 2021.: 191-197.

AZATO, F. K. et al. Influência do tratamento das desordens temporomandibulares na dor e na postura global. Revista Dor, v. 14, n. 4, p. 280-283, dez. 2013.

BONOTTO, et al. Viscossuplementação como tratamento das alterações internas da articulação temporomandibular: relato de casos. Revista Dor, v. 12, n. 3, p. 274-278, set. 2011.

BOULOUX, G. F. et al. Is hyaluronic acid or corticosteroid superior to lactated ringer solution in the short-term reduction of temporomandibular joint pain after arthrocentesis? Part 1. Journal of Oral and Maxillofacial Surgery, v. 75, n. 1, p. 52-62, 2017.

DANTAS, L. S. et al. A evolução do tratamento das desordens temporomandibulares. Braz J Surg Clin Res, v. 27, n. 3, p. 108-113, 2019.

DE RIU, G. at al. Arthrocentesis and temporomandibular joint disorders: clinical and radiological results of a prospective study. Int J Dent, doi: 10.1155/2013/790648. Epub 2013 Nov 11.

GIRADDI, G. B. et al. Internal derangement of temporomandibular joint: an evaluation of effect of corticosteroid injection compared with injection of sodium hyaluronate after arthrocentesis. Journal of maxillofacial and oral surgery, v. 11, n. 3, p. 258-263, 2012.

GROSSMANN, E. Arthrocentesis techniques applied to arthrogenic temporomandibular joint disorders. Rev Dor. São Paulo, Porto Alegre, RS, p. 374-381, dez. 2012.

LEE, H. S. et al. Effect of simultaneous therapy of arthrocentesis and occlusal splints on temporomandibular disorders: anterior disc displacement without reduction. Journal of the Korean Association of Oral and Maxillofacial Surgeons, v. 39, n. 1, p. 14-20, 2013.

MACIEL, L. F. O.; LANDIM, F. S.; VASCONCELOS, B. C. Otological findings and other symptoms related to temporomandibular disorders in young people. British Journal of Oral and Maxillofacial Surgery, v. 56, n. 8, p. 739-743, 2018.

MATSA, S. et al. Temporomandibular joint arthrocentesis for closed lock - A prospective analysis of 10 consecutive cases. Univer J of Dent Scienc, v. 1, n. 3, p. 225-229, 2010.

ROSSINI, R. et al. Double-Needle Arthrocentesis with Viscosupplementation in Patients with Temporomandibular Joint Disc Displacement without Reduction. Clinics (São Paulo, Brazil), v. 76, e2840, 2021. 
SCHIFFMAN, E. et al. Diagnostic Criteria for Temporomandibular Disorders (DC/TMD) for Clinical and Research Applications: recommendations of the International RDC/TMD Consortium Network and Orofacial Pain Special Interest Group. J Oral Facial Pain Headache, v. 28, n. 1, p. 6-27, 2014.

SILVA, H. B; SOARES, J. L. Análise da abordagem fisioterapêutica no tratamento da disfunção temporomandibular: revisão integrativa. Revista Eletrônica Acervo Saúde, n. 19, p. 210, dez 2018.

STEPAN, L.; SHAW, C.L.; OUE, S. Temporomandibular disorder in otolaryngology: systematic review. J Laryngol Otol, v. 131, n. 1, p. 50-56, 2017.

TAN, D. B. P.; KRISHNASWAMY, G. A Retrospective Study of Temporomandibular Joint Internal Derangement Treated with Arthrocentesis and Arthroscopy. Proceedings of Singapore Healthcare, v. 21, n. 1, p. 73-78, 2012.

URBANO, E. S. et al. Avaliação da eficácia da artrocentese associada à viscossuplementação no tratamento das desordens temporomandibulares: relato de caso. HU Revista, v. 46, p. 1-8, 2020. 